\title{
A Facile Synthesis of 1,3,7-Trihydroxyxanthone and its Regioselective Coupling Reactions with Prenal: Simple and Efficient Access to Osajaxanthone and Nigrolineaxanthone F
}

\author{
Mukulesh Mondal, ${ }^{a}$ Vedavati G. Puranik ${ }^{b}$ and Narshinha P. Argade ${ }^{a, *}$ \\ ${ }^{a}$ Division of Organic Chemistry (Synthesis), ${ }^{b}$ Centre for Material Characterization, \\ National Chemical Laboratory, Pune 411 008, India \\ np.argade@ncl.res.in
}

SI-1

Table of contents in Supporting Information

\begin{tabular}{|c|c|}
\hline Sr. No & Contents \\
\hline SI-2 & Experimental: General Description \\
\hline SI-3 & ${ }^{1} \mathrm{H}$ spectra of compounds $\mathbf{3}$ and $\mathbf{4}$ \\
\hline SI-4 & ${ }^{13} \mathrm{C}$ and DEPT spectra of compound 4 \\
\hline SI-5 & ${ }^{1} \mathrm{H}$ and ${ }^{13} \mathrm{C}$ spectra of compound $\mathbf{5}$ \\
\hline SI-6 & DEPT spectra of compound 5 and ${ }^{1} \mathrm{H}$ spectra of compound $\mathbf{6}$ \\
\hline SI-7 & ${ }^{13} \mathrm{C}$ and DEPT spectra of compound $\mathbf{6}$ \\
\hline SI-8 & ${ }^{1} \mathrm{H}$ and ${ }^{13} \mathrm{C}$ spectra of compound 7a \\
\hline SI-9 & DEPT spectra of compound $\mathbf{7 a}$ and ${ }^{1} \mathrm{H}$ spectra of compound $\mathbf{7 b}$ \\
\hline SI-10 & ${ }^{13} \mathrm{C}$ and DEPT spectra of compound $\mathbf{7 b}$ \\
\hline SI-11 & ${ }^{1} \mathrm{H}$ and ${ }^{13} \mathrm{C}$ spectra of compound $7 \mathrm{c}$ \\
\hline SI-12 & DEPT spectra of compound $\mathbf{7 c}$ and ${ }^{1} \mathrm{H}$ spectra of compound $\mathbf{8 a}$ \\
\hline SI-13 & ${ }^{13} \mathrm{C}$ and DEPT spectra of compound $\mathbf{8 a}$ \\
\hline SI-14 & ${ }^{1} \mathrm{H}$ spectra of compounds $\mathbf{8 b}$ and $\mathbf{8 c}$ \\
\hline SI-15 & Experimental section of single crystal X-ray crystallographic studies of compound 7a \\
\hline SI-16 & $\begin{array}{l}\text { Experimental section of single crystal X-ray crystallographic studies of compound } \mathbf{8 a} \\
\text { and ORTEP diagrams of compounds 7a and } \mathbf{8 a}\end{array}$ \\
\hline SI-17 & Packing data for compound 7a \\
\hline SI-18 & Packing data for compound $\mathbf{8 a}$ \\
\hline SI-19 & Preparation details \& tabulated analytical and spectral data of compounds $\mathbf{7 b} \& \mathbf{7 c}$ \\
\hline SI-20 & Preparation details \& tabulated analytical and spectral data of compounds $8 \mathbf{b} \&$ 8c \\
\hline
\end{tabular}




\section{SI-2}

\section{Experimental: General Description}

Commercially available 1,3,5-trimethoxybenzene, NBS, methyl 2,5-dihydroxybenzoate, benzylbromide, $10 \% \mathrm{Pd}-\mathrm{C}, \mathrm{BBr}_{3}$, 3-methyl-2-butenal, crotonaldehyde and citral were used. Melting points are uncorrected. Dichloromethane was distilled from $\mathrm{CaH}_{2}$ under argon. Tetrahydrofuran was freshly distilled from benzophenone ketyl radical under argon prior to use. Column chromatographic separations were carried out on ACME silica gel (60-120 mesh). All yields given refer to as isolated yields. IR spectra were recorded on FT-IR spectrometer. MS experiments were performed on a low resolution magnetic sector mass spectrometer. 


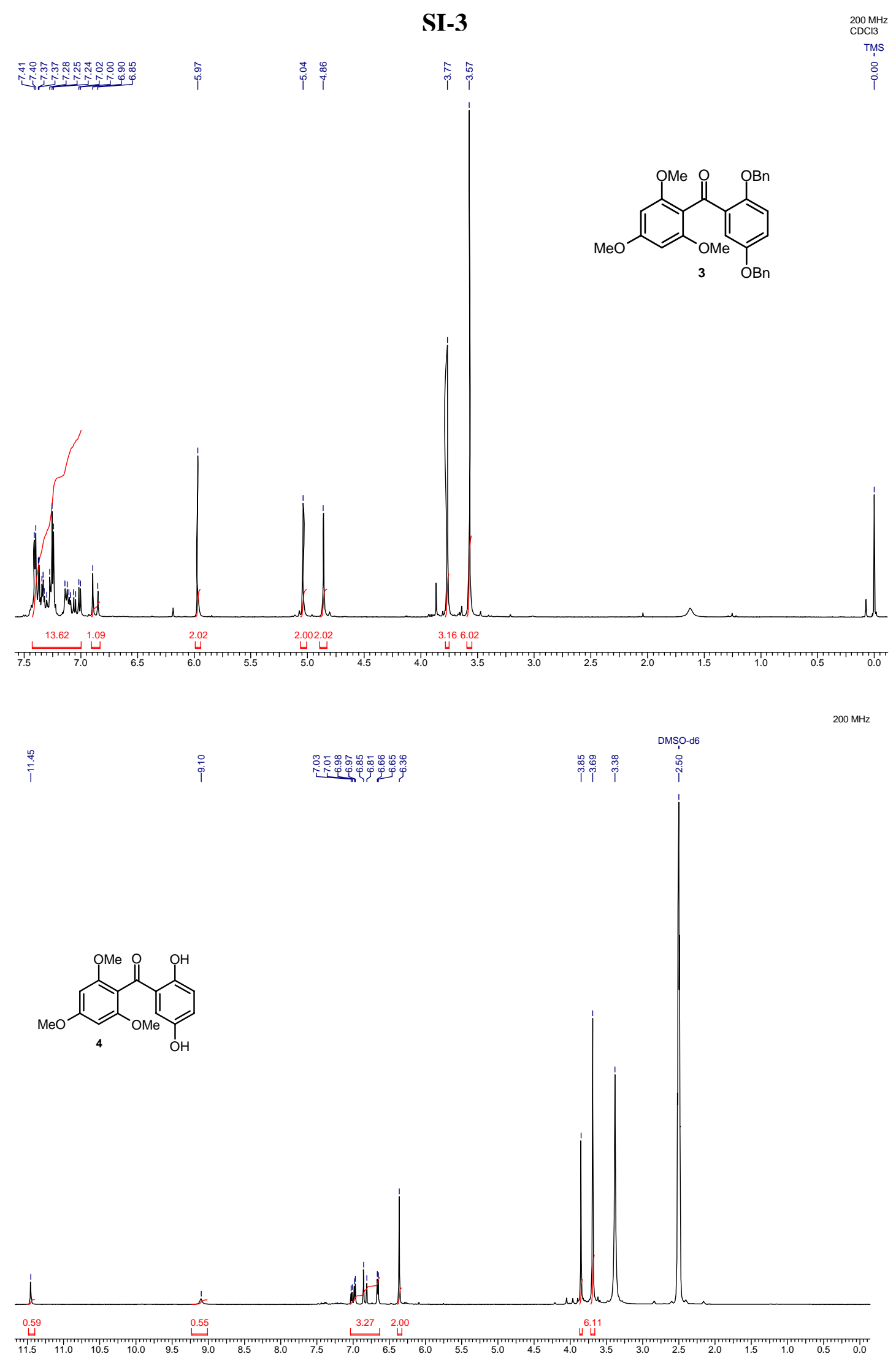




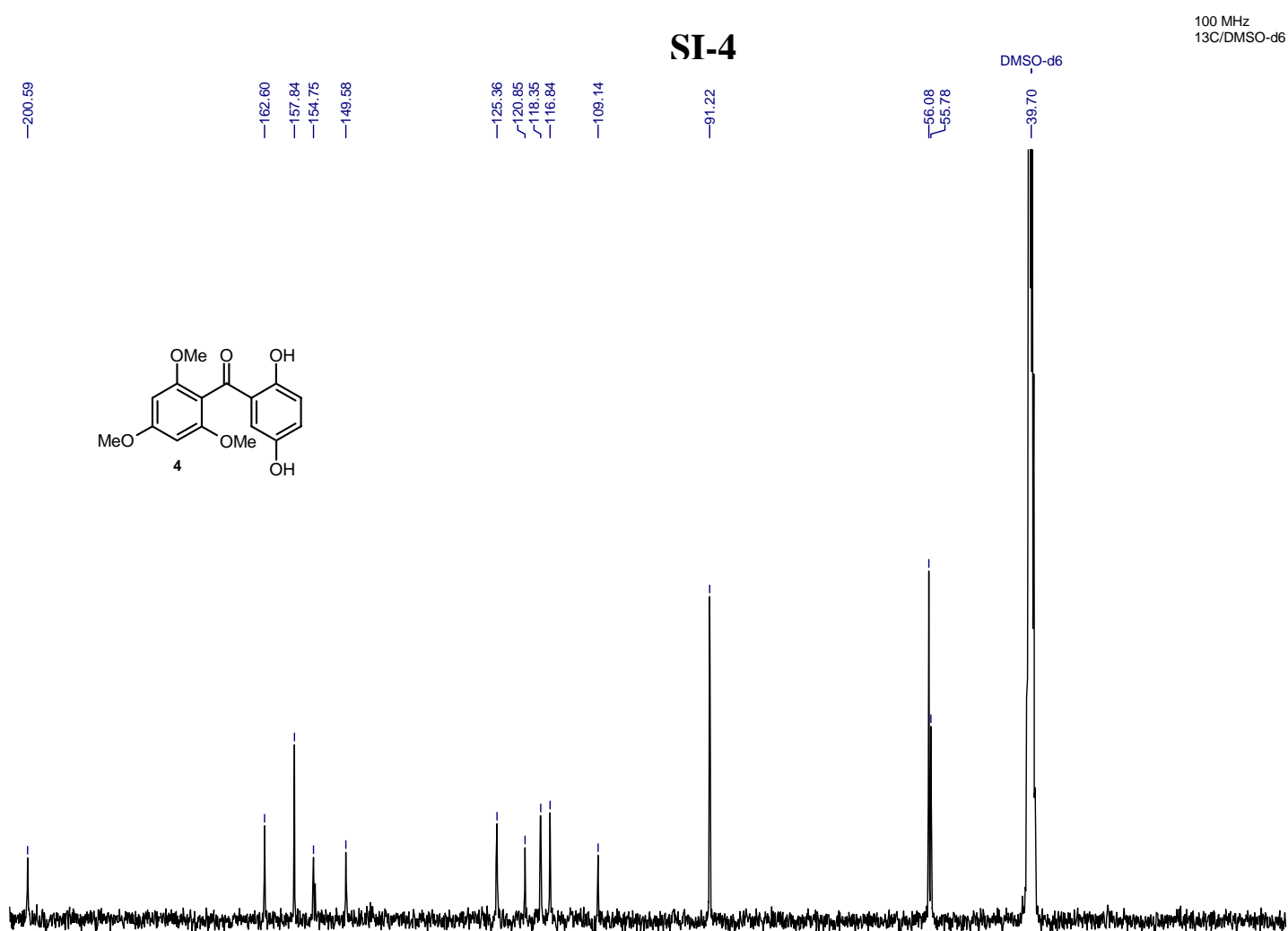

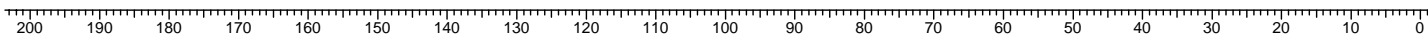

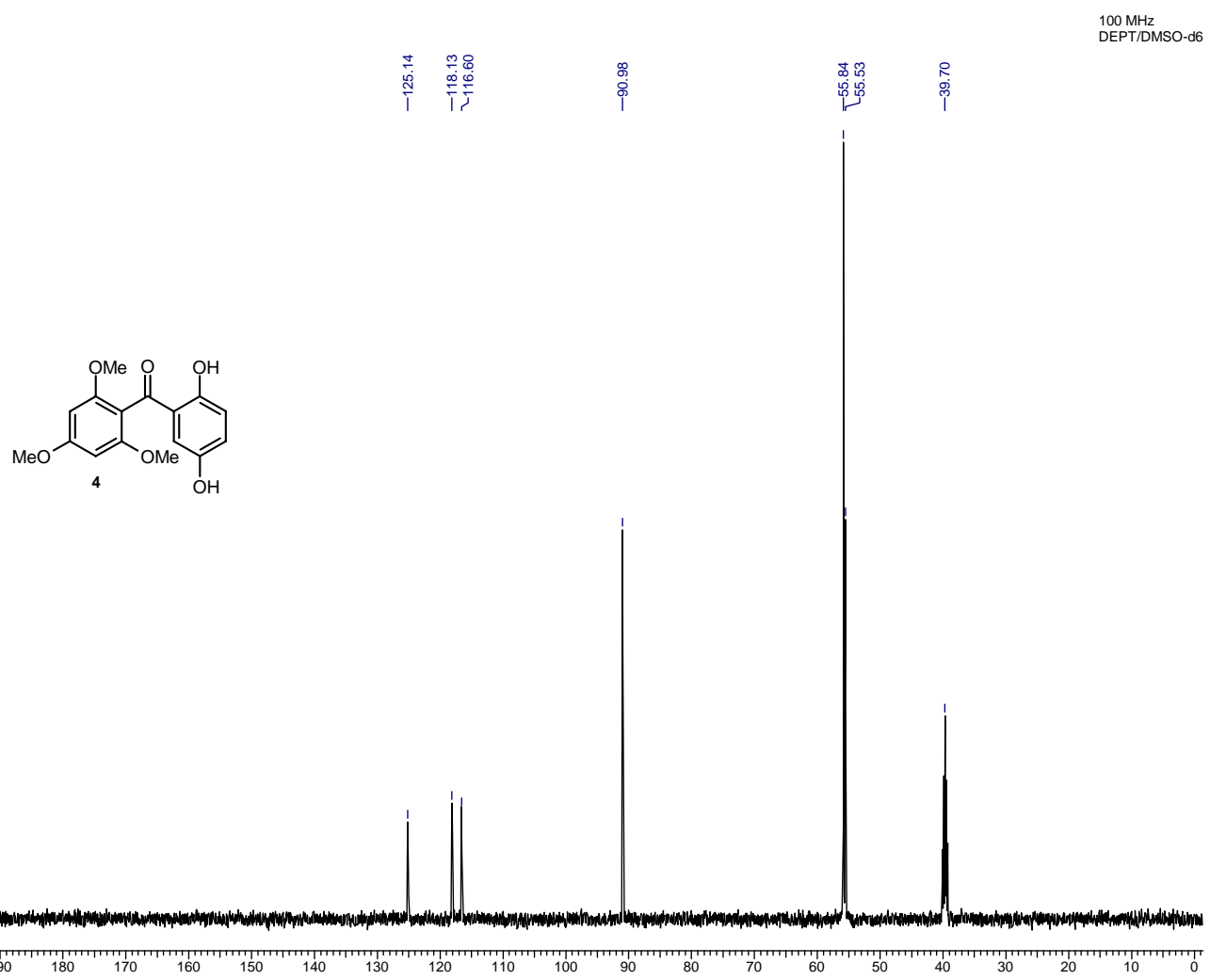


SI-5

$200 \mathrm{MHz}$
DMSO-d6

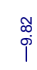

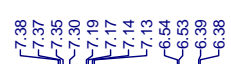

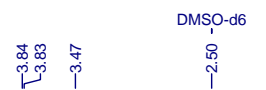

$\overbrace{\mathrm{MeO}}^{\mathrm{OMe}}$

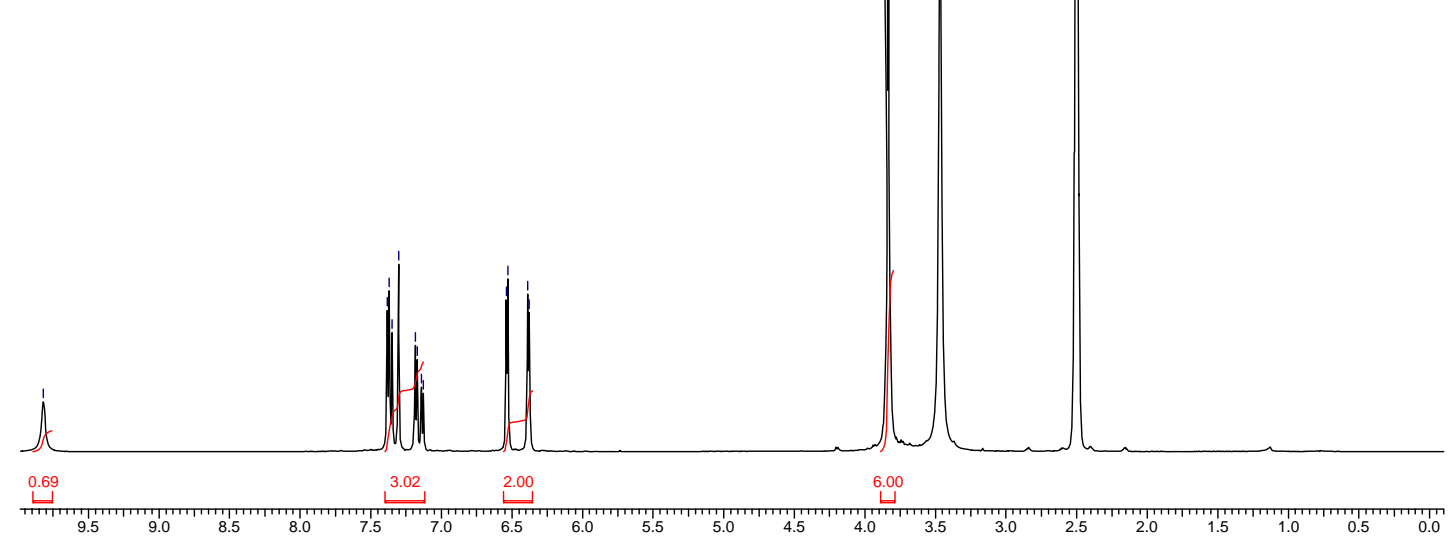<smiles>COc1cc(OC)c2c(=O)c3cc(O)ccc3oc2c1</smiles>

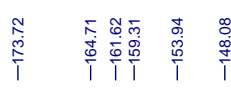

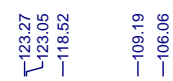

畓兽

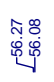

$50 \mathrm{MHz}$

3C/DMSO-d6

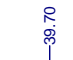

1
0
0
0
0
0
0

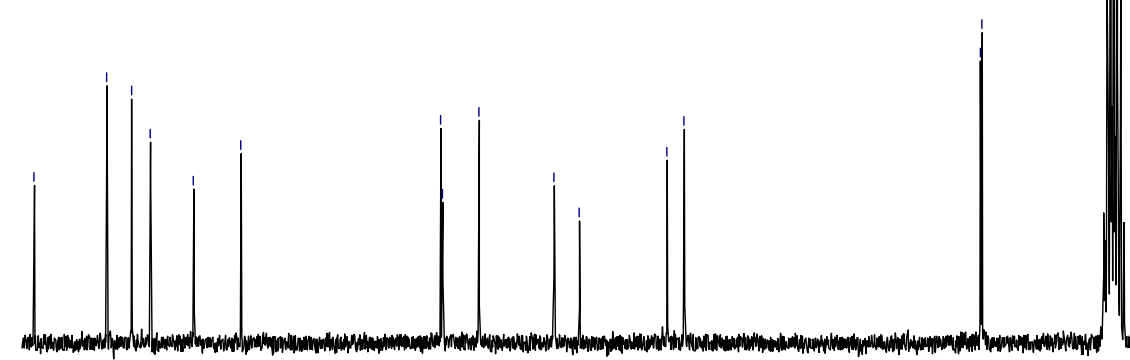

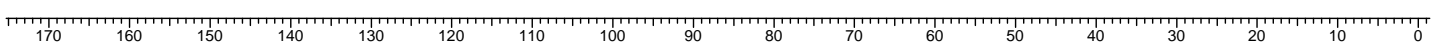


SI-6

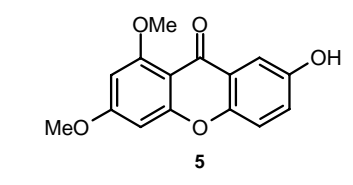

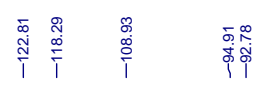

Do MEHT

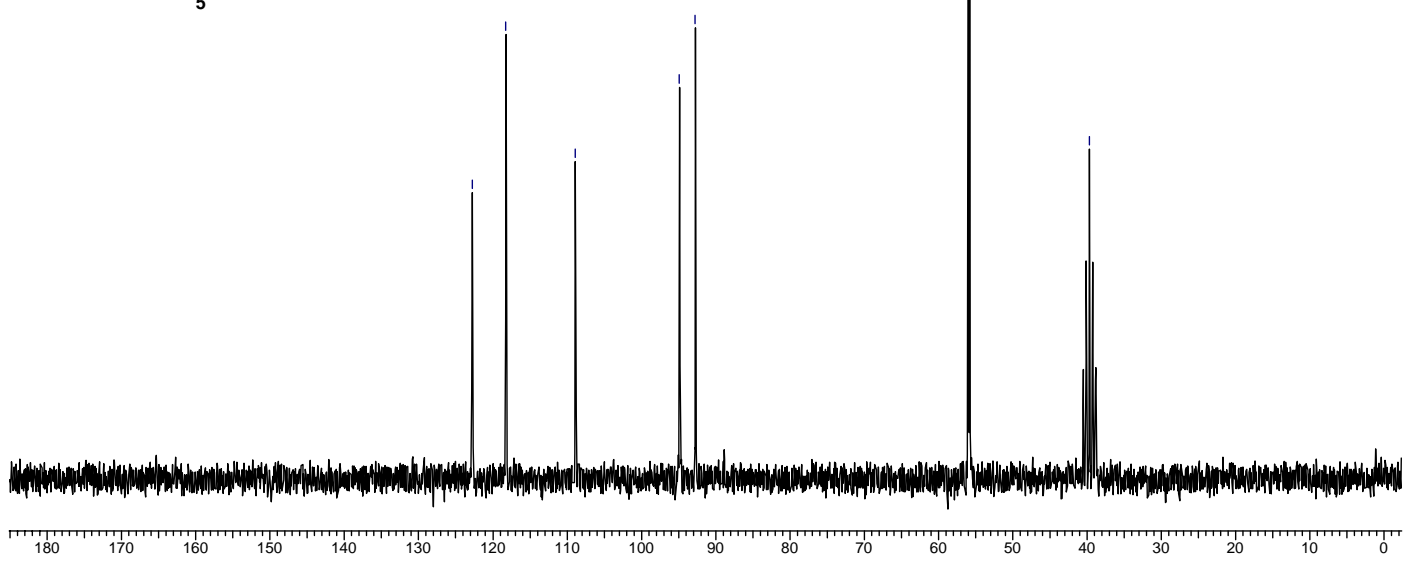

$\stackrel{\mathbb{\Xi}}{T}$

章

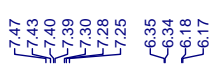

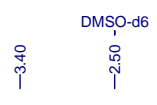

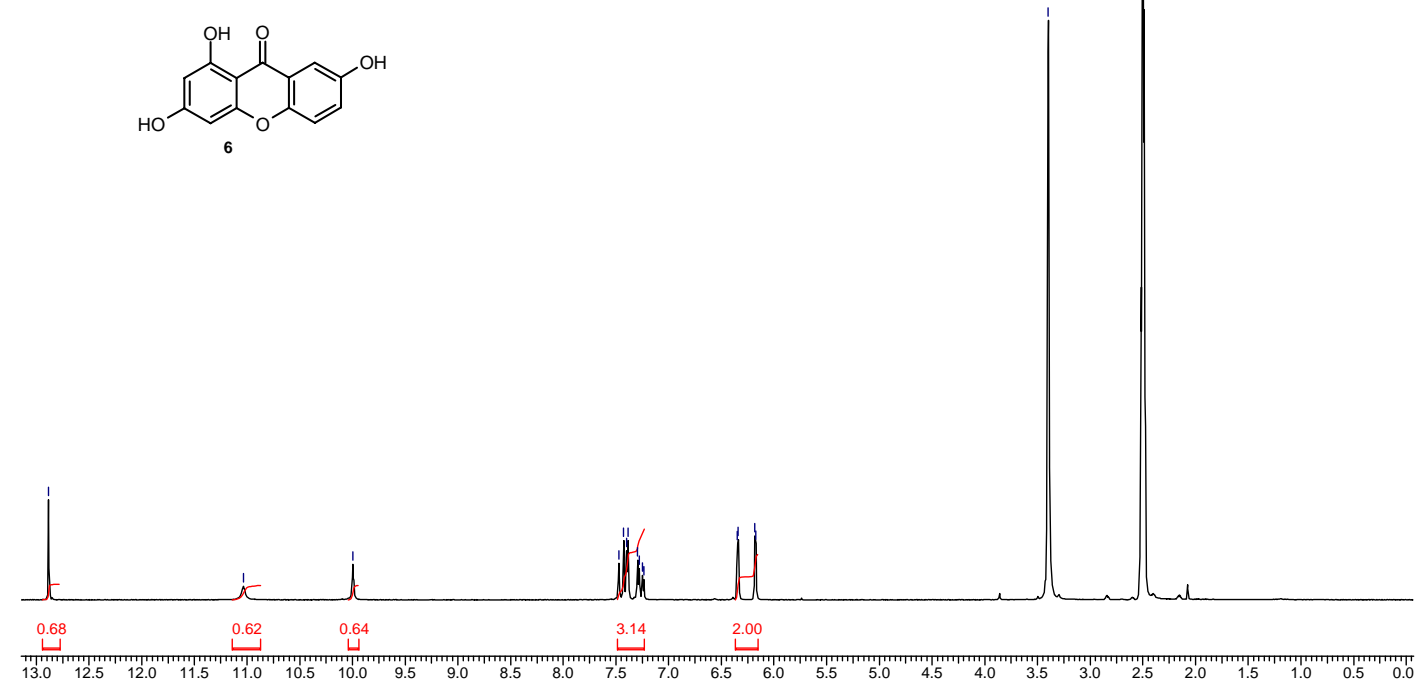

$200 \mathrm{MHz}$

DMSO-d6 

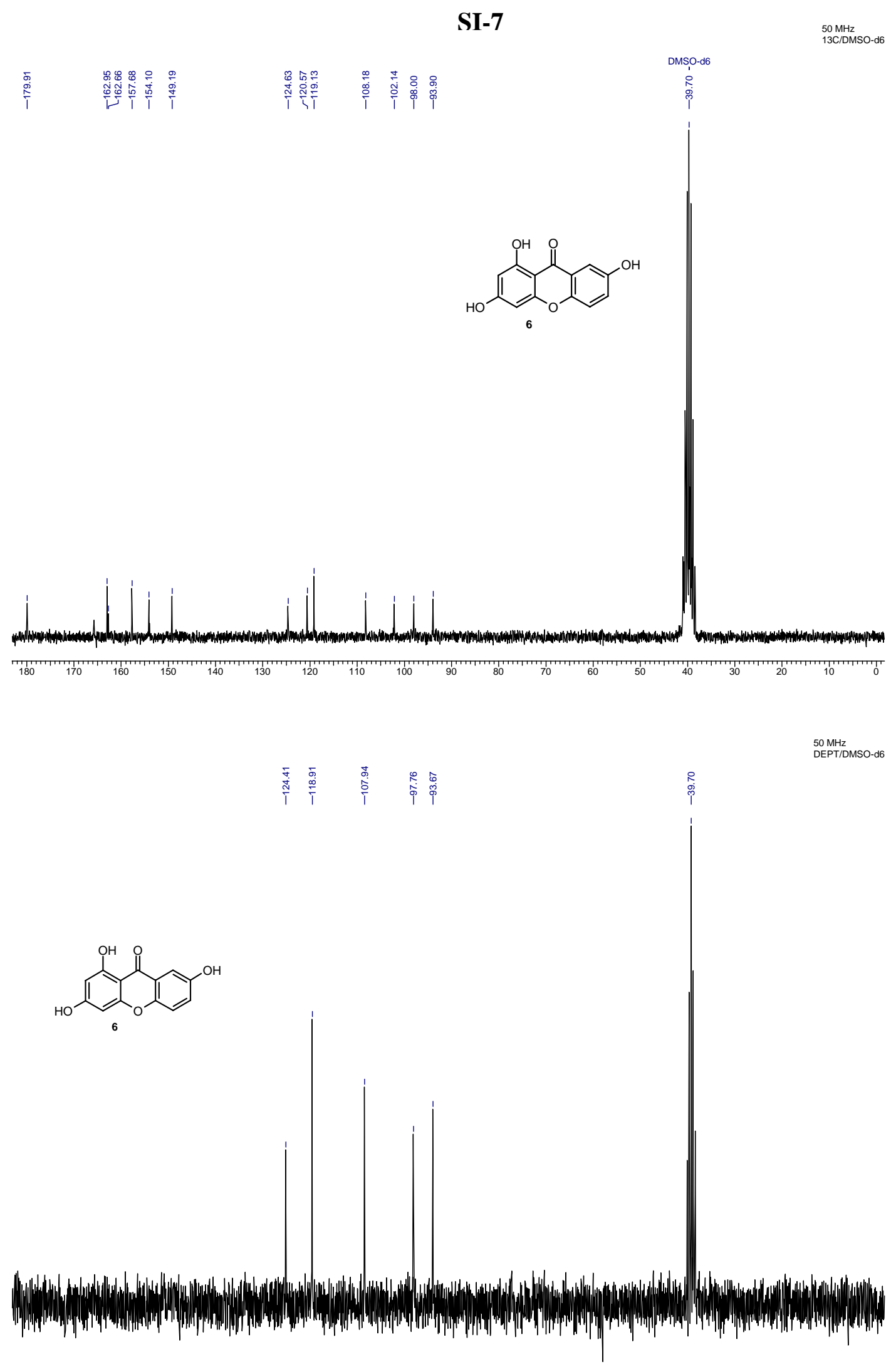

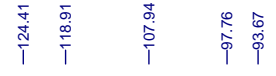

?.

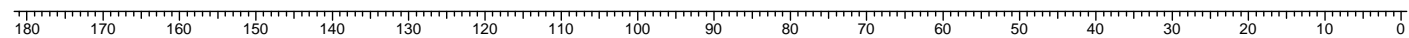



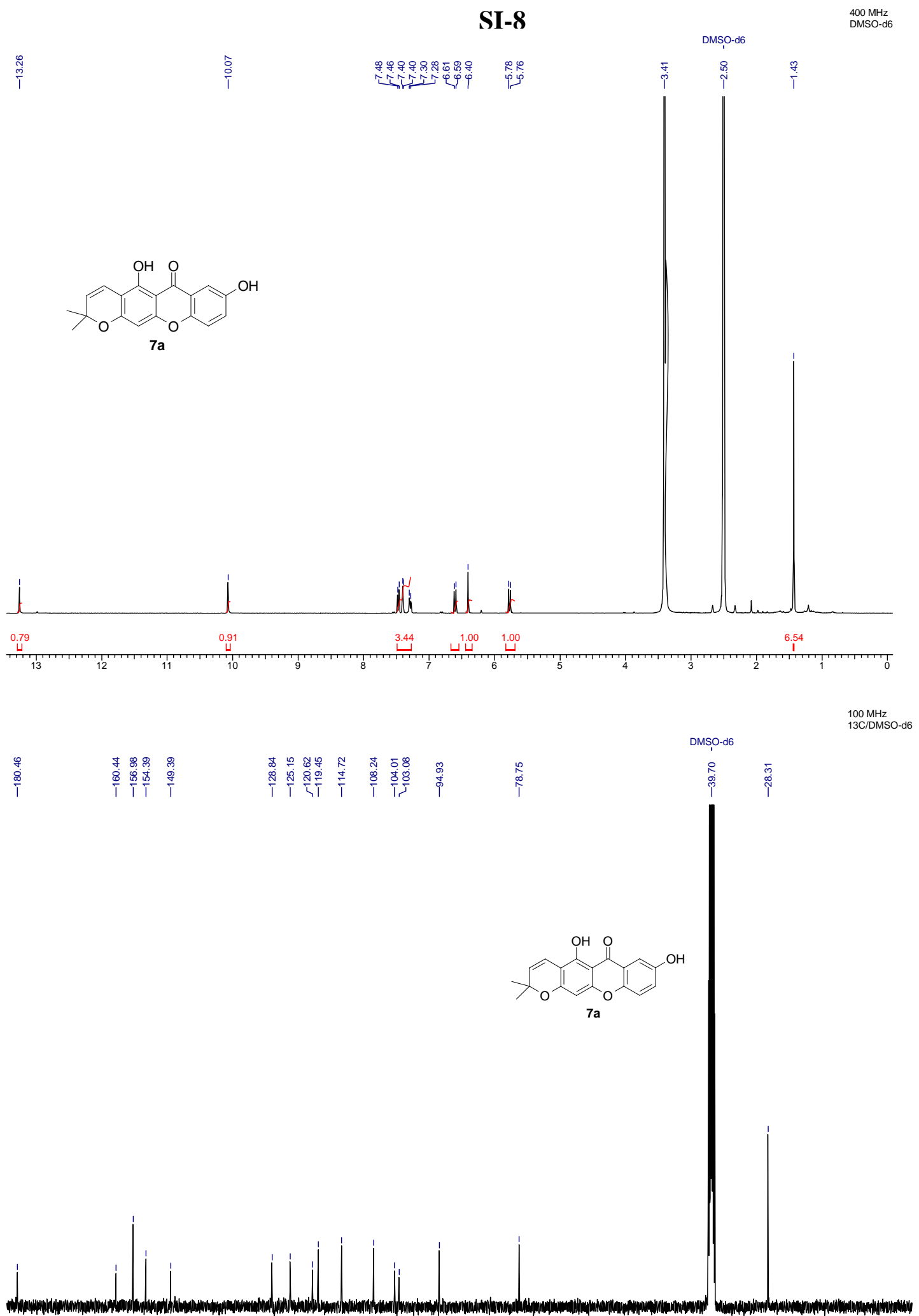


\section{SI-9}

$100 \mathrm{MHz}$

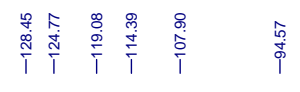
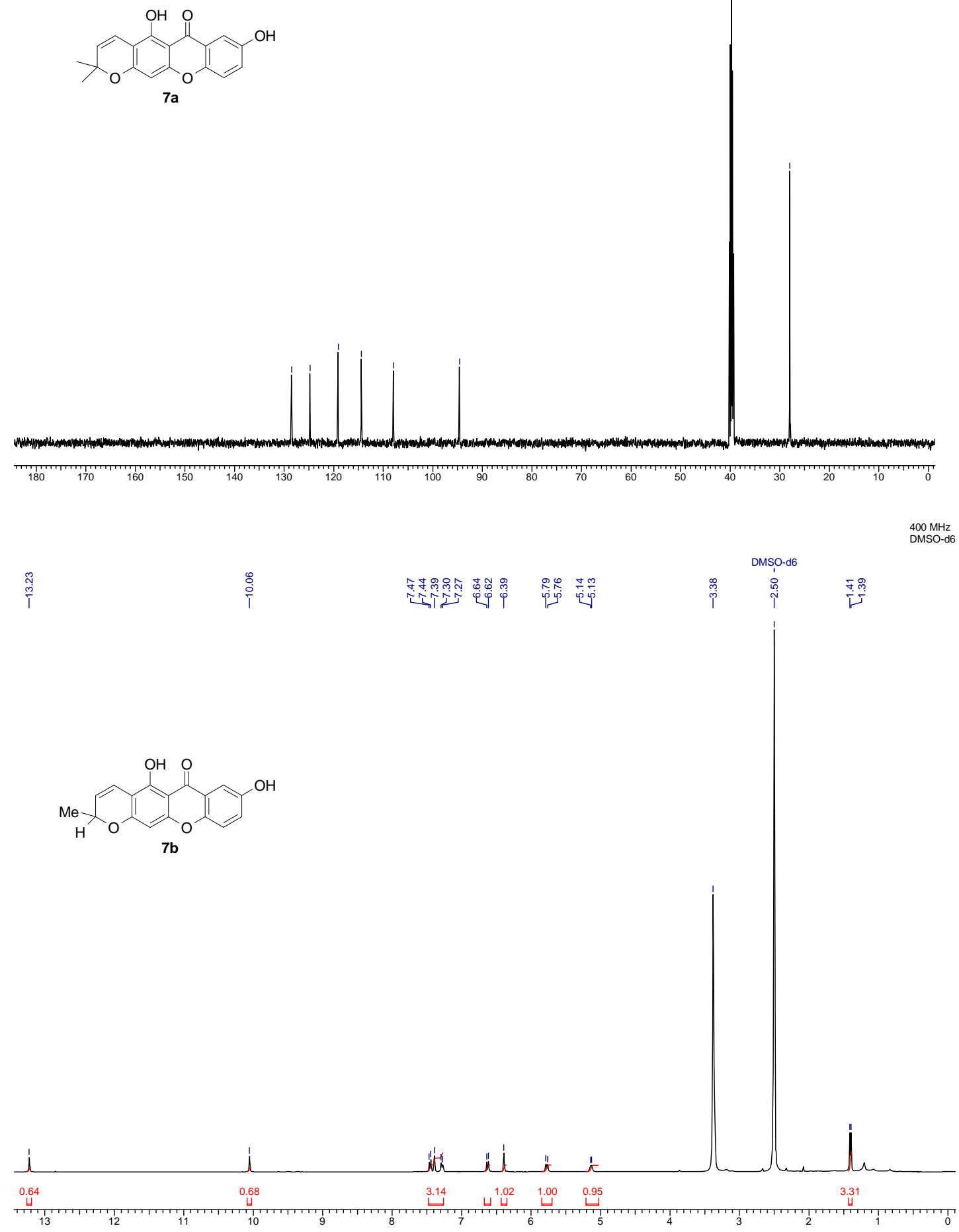

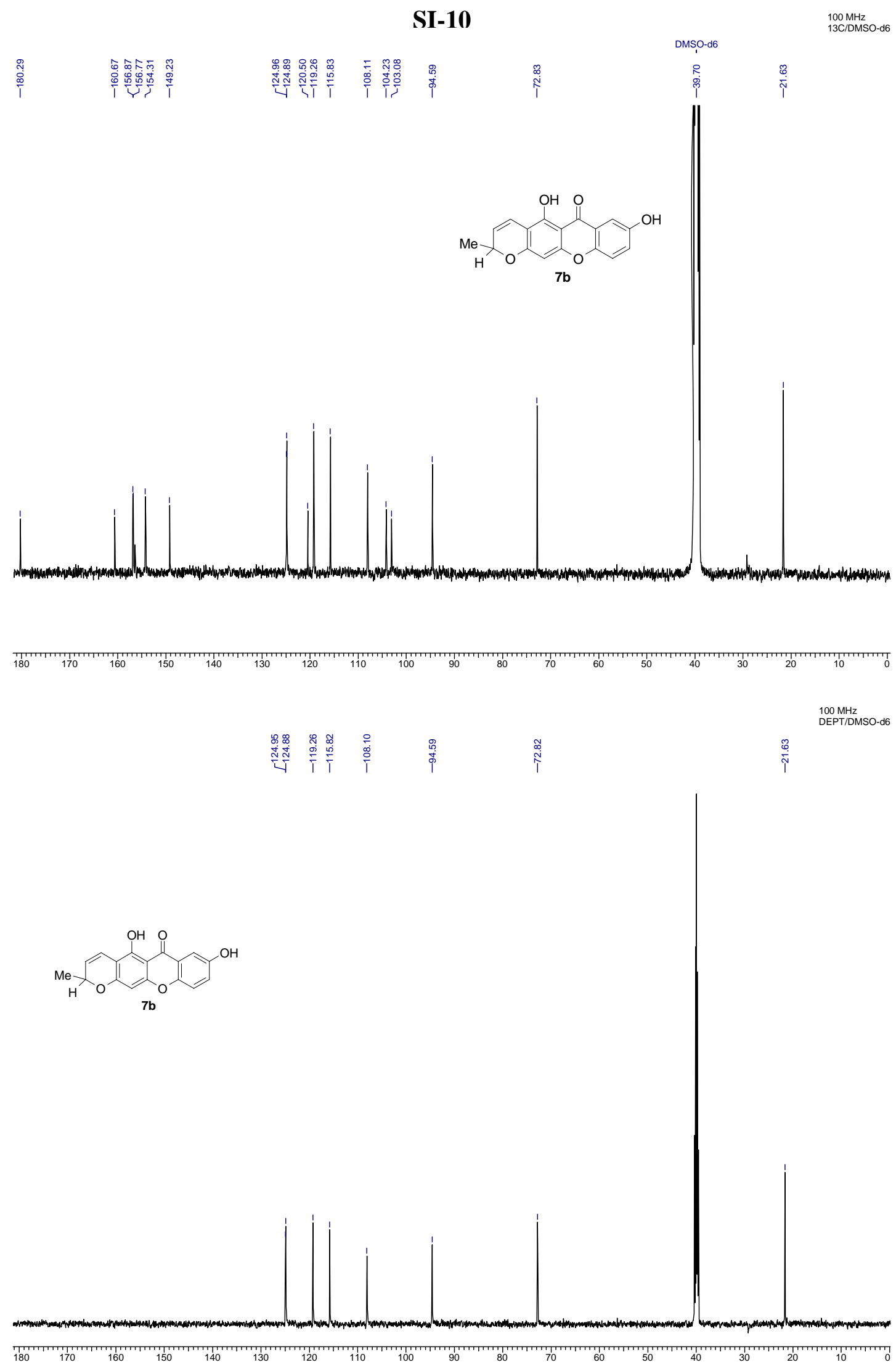

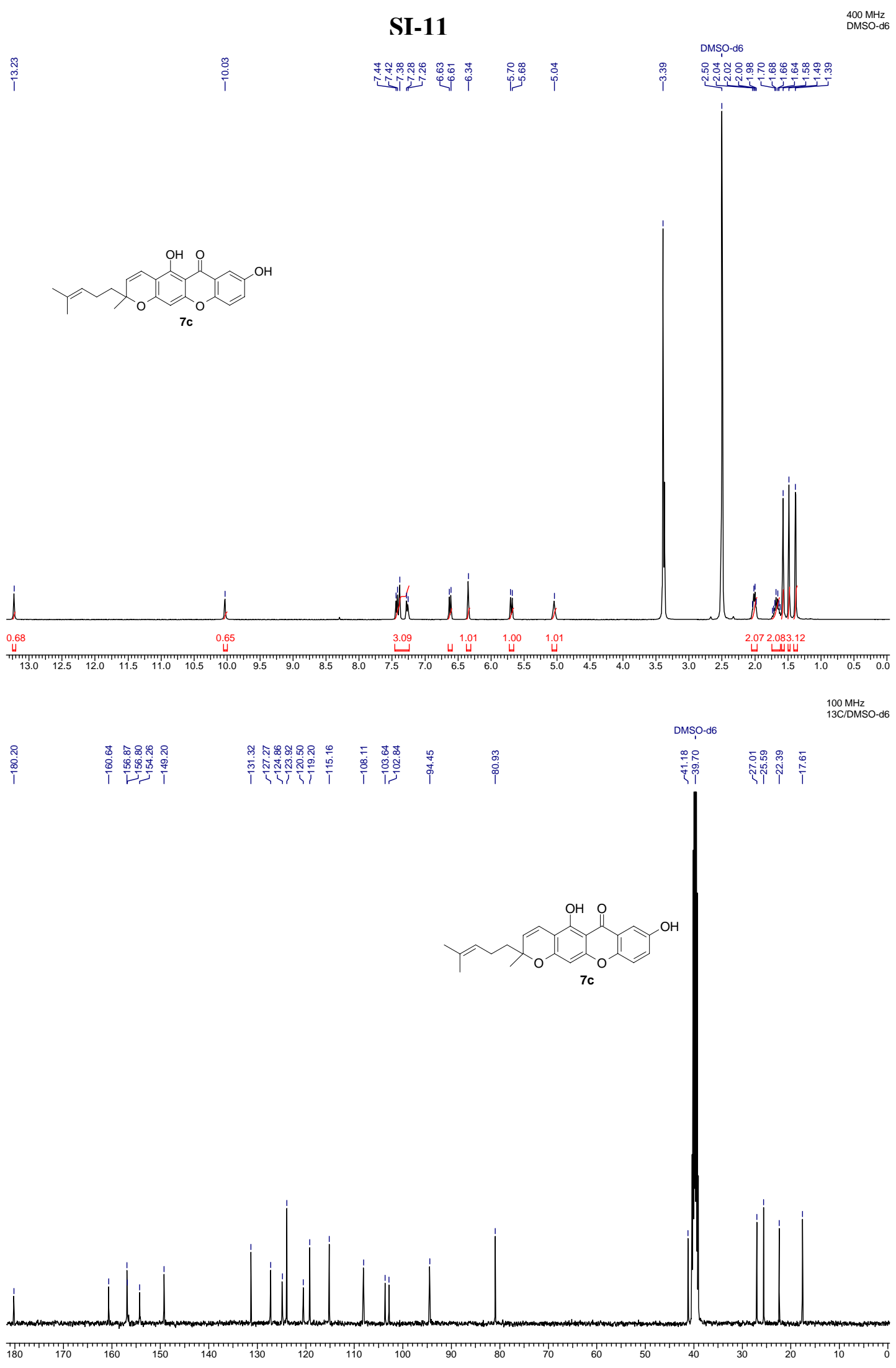


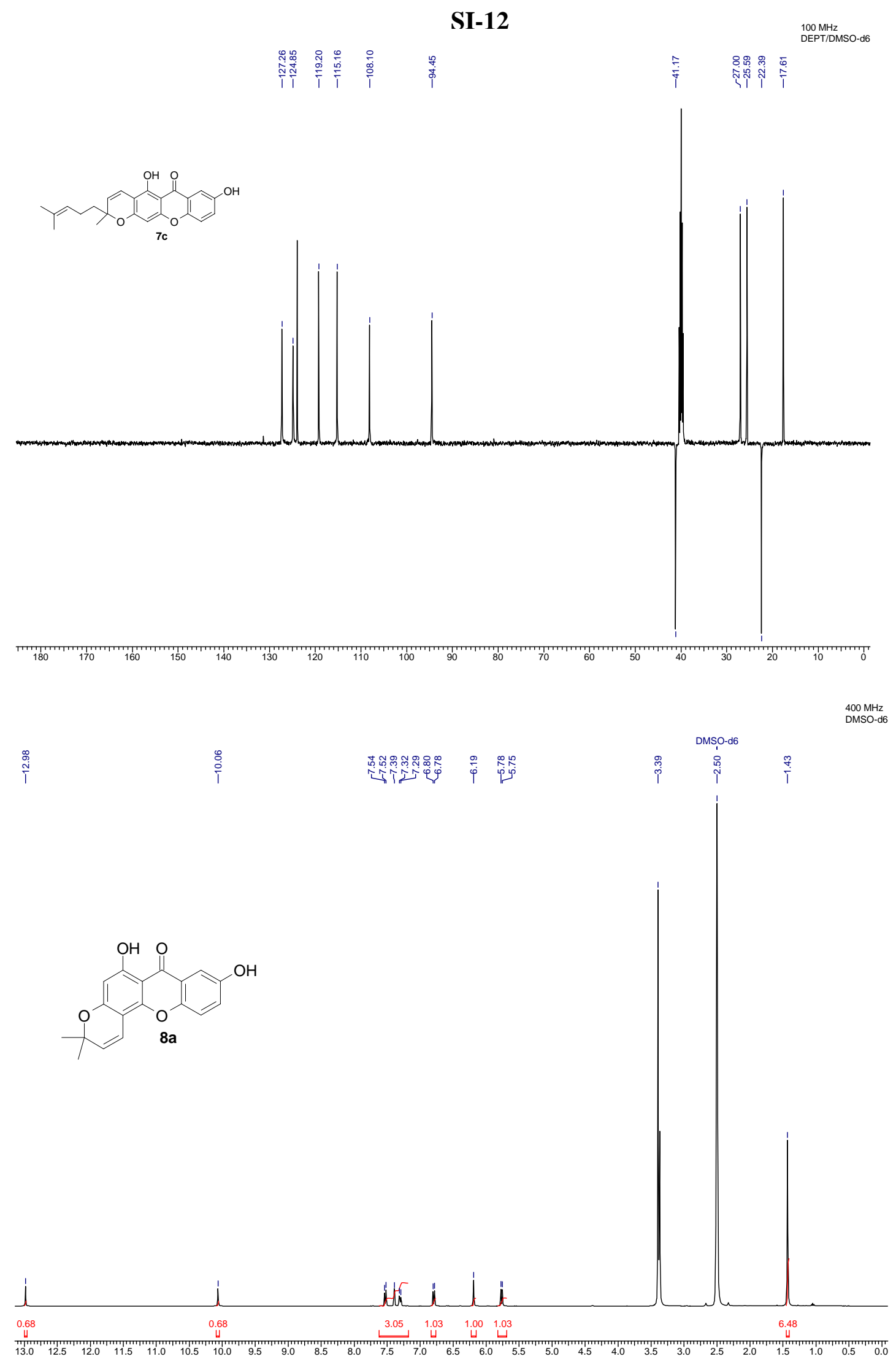



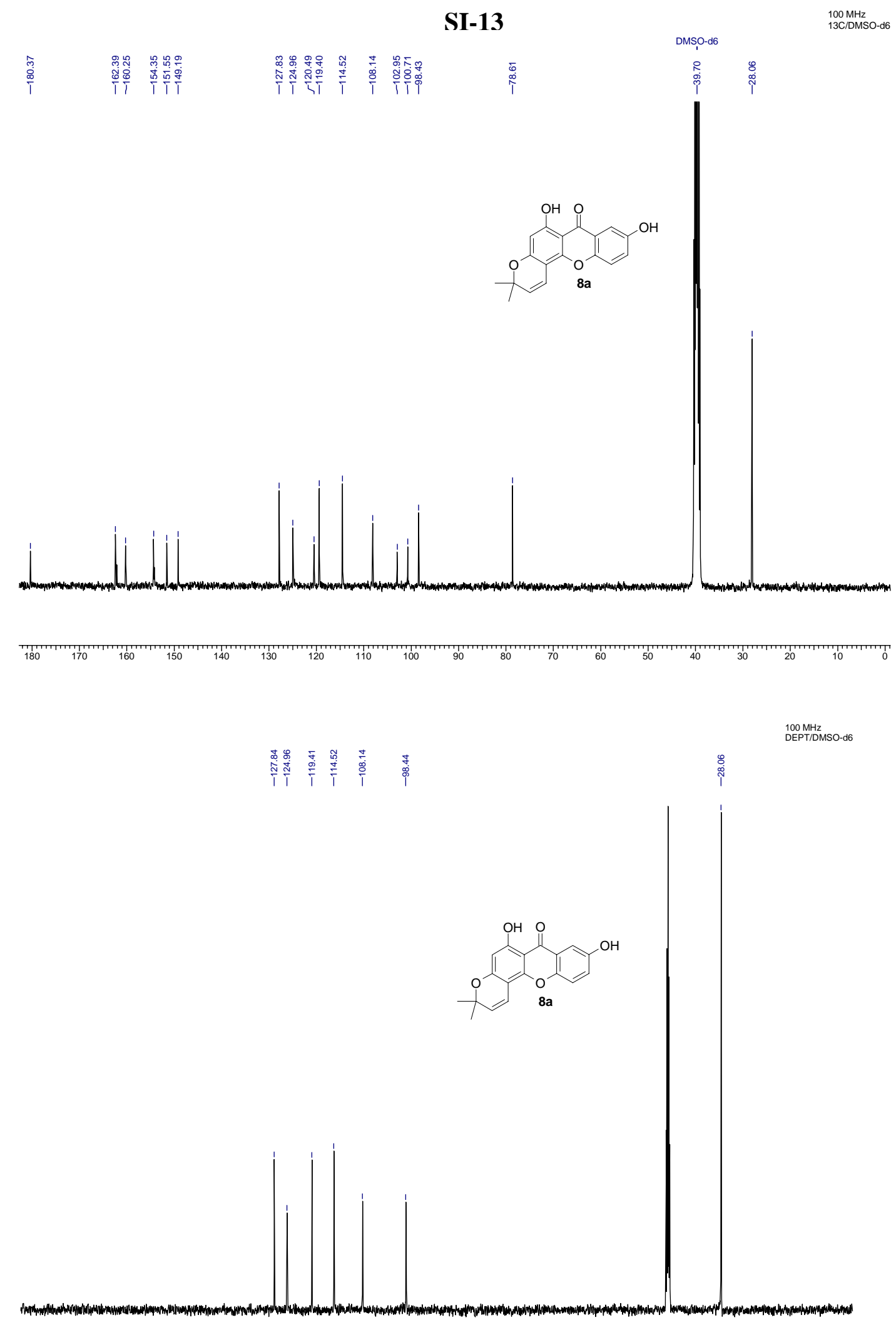

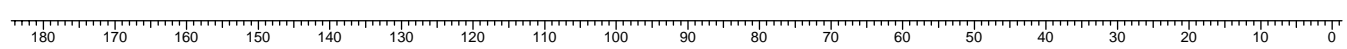




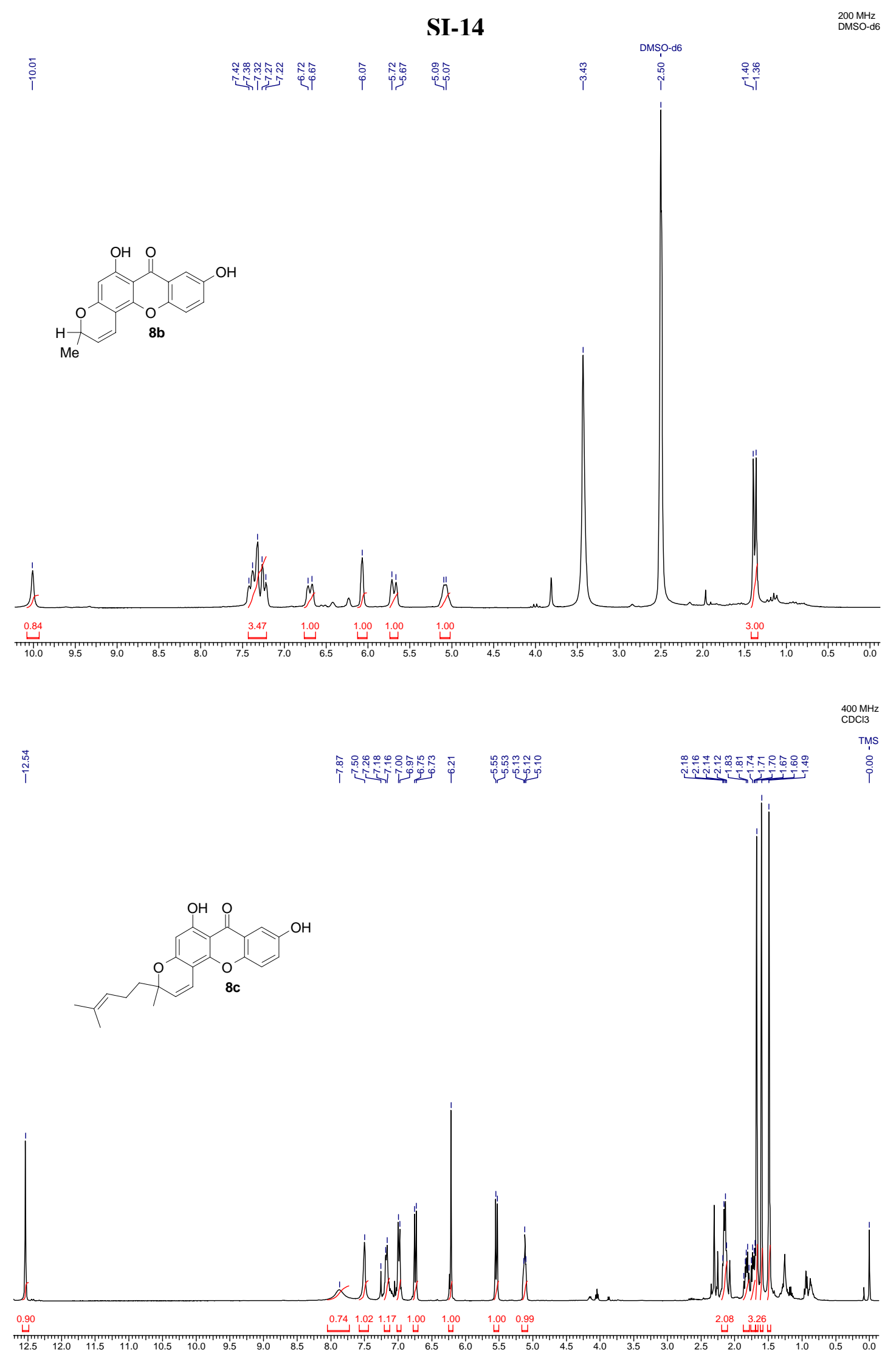




\section{SI-15}

\section{Experimental Section}

\section{Single Crystal X-ray Crystallographic Studies:}

The X-ray data of 7a and 8a were collected on a SMART APEX CCD single crystal Xray diffractometer with omega and phi scan mode and different number of scans and exposure times for different crystals using $\lambda \mathrm{MoK}_{\alpha}=0.71073 \AA$ radiation, at $\mathrm{T}=293(2)$ $\mathrm{K}$ with Oscillation $/$ frame $-0.3^{\circ}$, maximum detector swing angle $=-30.0^{\circ}$, beam center $=$ (260.2, 252.5), in plane spot width $=1.24$. All the data were corrected for Lorentzian, polarization and absorption effects using SAINT and SADABS programs. The crystal structures were solved by direct method using SHELXS-97 and the refinement was performed by full matrix least squares of $F^{2}$ using SHELXL-97 (G. M. Sheldrick, SHELX-97 program for crystal structure solution and refinement, University of Göttingen, Germany, 1997).

Crystal Data for 7a $\left(\mathrm{C}_{18} \mathrm{H}_{14} \mathrm{O}_{5}\right): M=310.29$, Single crystals of the compound were grown by slow evaporation of the solution mixture of 1:1 methanol and ethanol. Yellow colored needle of approximate size $0.27 \times 0.18 \times 0.06 \mathrm{~mm}$ for data collection, hemisphere data acquisition. Total scans $=3$, total frames $=1271$, exposure $/$ frame $=7.0 \mathrm{sec} /$ frame, $\theta$ range $=2.78$ to $24.99^{\circ}$, completeness to $\theta$ of $24.99^{\circ}$ is $98.4 \%$, monoclinic, space group $\mathrm{P} 21 / \mathrm{c}, a=11.8863(14), b=5.3819(7), c=\mathrm{c}=22.632(3) \AA, \beta=101.436(2){ }^{\circ}, V=$ 1419.0(3) $\AA^{3}, Z=4, D_{c}=1.452 \mathrm{mg} \mathrm{m}^{-3}, \mu\left(\right.$ Mo $\left.\mathrm{K}_{\alpha}\right)=0.107 \mathrm{~mm}^{-1}, 6315$ reflections measured, 2461 unique [I $>2 \sigma(\mathrm{I})]$, $\mathrm{R}$ value 0.0338 , wR2 $=0.0724$ 


\section{SI-16}

Crystal Data for $\mathbf{8 b}\left(\mathrm{C}_{18} \mathrm{H}_{14} \mathrm{O}_{5}\right): M=264.29$, Single crystals of the compound were grown by slow evaporation of the solution in ethanol. Yellow colored crystal of size 0.48 x 0.33 x $0.31 \mathrm{~mm}$, multi scan data acquisition. Total scans $=3$, total frames $=1715$, exposure $/$ frame $=20.0 \mathrm{sec} /$ frame, $\theta$ range $=2.22$ to $25.00^{\circ}$, completeness to $\theta$ of $25.0^{\circ}$ is $99.8 \%$. monoclinic, space group C2/c, $a=18.5994$ (9), $b=10.6620$ (5), c 14.7627(7) $\AA, \beta=102.537(1)^{\circ}, V=2857.7(2) \AA^{3}, Z=8, D_{c}=1.442 \mathrm{mg} \mathrm{m}^{-3}, \mu\left(M_{\alpha K}\right)=0.106 \mathrm{~mm}^{-}$ ${ }^{1}$, 9421 reflections measured, 2515 unique [I>2 $\left.\sigma(\mathrm{I})\right]$, R value 0.0424 , wR2 $=0.1095$.

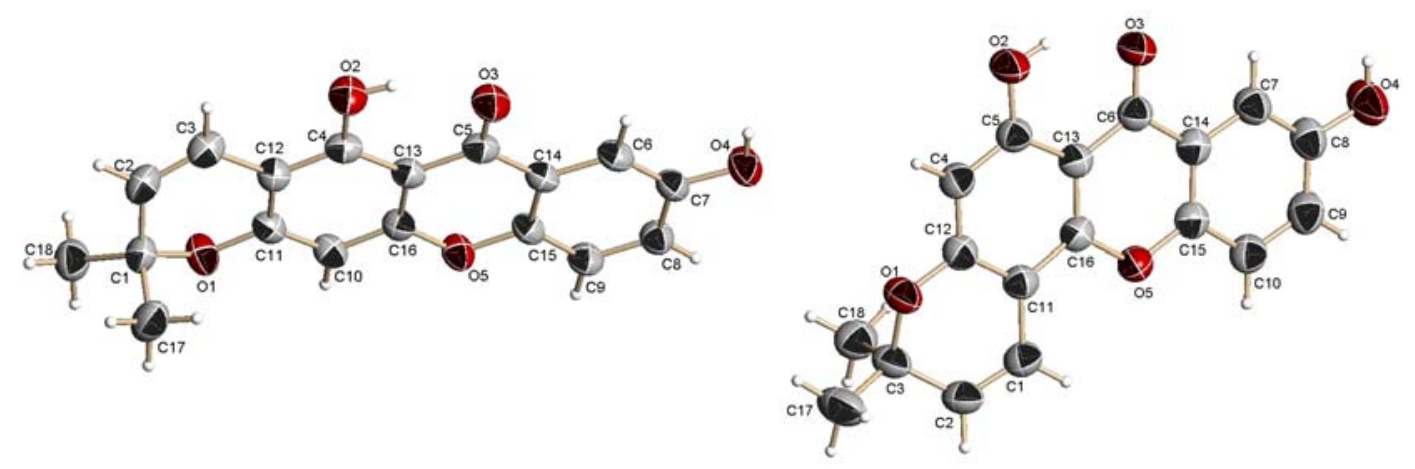

ORTEP Diagram of 7a.

ORTEP Diagram of 8a.

(Both the ellipsoids are drawn at 50\% probability). 


\section{SI-17}

Both intramolecular O-H....O hydrogen bonding and intermolecular O-H....O hydrogen bondings is seen for the compound 7a (Figure 1). The molecules of 7a pack in a zigzag manner when viewed down “a”-axis (Figure 1)

Analysis of Potential Hydrogen Bonds (7a)

\begin{tabular}{|c|c|c|c|}
\hline Donor --- H....Acceptor & H...A & D...A & D - H...A \\
\hline $\mathrm{O}(2)$-- $\mathrm{H}(2) \quad . . \mathrm{O}(3)^{\text {Intra }}$ & 1.88 & $2.604(2)$ & 147 \\
\hline $\mathrm{O}(4)--\mathrm{H}(4) \quad . . \mathrm{O}(3)^{\mathrm{i}}$ & 1.92 & $2.722(2)$ & 166 \\
\hline
\end{tabular}

Equivalent Position Code

${ }^{\mathrm{i}}=-\mathrm{x}, 1 / 2+\mathrm{y}, 1 / 2-\mathrm{z}$ 


\section{SI-18}

Molecule 8a exists as a dimer by intramolecular O-H.... hydrogen bonding and intermolecular $\mathrm{O}-\mathrm{H} . . . \mathrm{O}$ hydrogen bondings (Figure 2) in the packing of the molecules when viewed down “c”-axis (Figure 2).

Analysis of Potential Hydrogen Bonds (8a)

\begin{tabular}{|c|c|c|c|}
\hline Donor --- H....Acceptor & H...A & D...A & D - H...A \\
\hline $\mathrm{O}(2) \quad--\mathrm{H}(2) \quad . . \mathrm{O}(3)^{\text {intra }}$ & 1.89 & $2.614(2)$ & 147 \\
\hline $\mathrm{O}(4)--\mathrm{H}(4) \quad . . \mathrm{O}(3)^{\mathrm{i}}$ & 2.02 & $2.772(2)$ & 152 \\
\hline
\end{tabular}

Equivalent Position Code

$====================$
$\quad=-x, 3-y,-z$ 


\section{SI-19}

5,8-Dihydroxy-2-methyl-2H,6H-pyrano[3,2-b]xanthen-6-one (7b). Compound 7b was prepared similarly using 6 (250 mg, $1.02 \mathrm{mmol})$ and crotonaldehyde $(0.40 \mathrm{~mL}, 5.12$ mmol). 7b: Yellow solid (200 mg, 66\%); mp 263-265 ${ }^{\circ} \mathrm{C} ;{ }^{1} \mathrm{H}$ NMR (DMSO-d $d_{6}, 400$ MHz) $\delta 1.40(\mathrm{~d}, J=6.6 \mathrm{~Hz}, 3 \mathrm{H}), 5.14(\mathrm{~m}, 1 \mathrm{H}), 5.78(\mathrm{dd}, J=10.0 \& 2.4 \mathrm{~Hz}, 1 \mathrm{H}), 6.39$ (s, 1H), 6.63 (d, $J=9.9 \mathrm{~Hz}, 1 \mathrm{H}), 7.29$ (dd, $J=8.8 \& 2.0 \mathrm{~Hz}, 1 \mathrm{H}), 7.39$ (d, $J=2.2 \mathrm{~Hz}$,

1H), $7.46(\mathrm{~d}, J=8.8 \mathrm{~Hz}, 1 \mathrm{H}), 10.06$ (s, $1 \mathrm{H}), 13.23(\mathrm{~s}, 1 \mathrm{H}) ;{ }^{13} \mathrm{C}$ NMR (DMSO-d 6,100 MHz) $\delta$ 21.6, 72.8, 94.6, 103.1, 104.2, 108.1, 115.8, 119.3, 120.5, 124.9, 125.0, 149.2, 154.3, 156.8, 156.9, 160.7, 180.3; MS (m/z) 297, 279, 264, 239, 217, 180, 149, 117; IR (Nujol) 3279, 1653, 1611, 1585, $1464 \mathrm{~cm}^{-1}$. Anal. Calcd for $\mathrm{C}_{17} \mathrm{H}_{12} \mathrm{O}_{5}$ : C, 68.92; H, 4.08. Found: C, 69.01; H, 3.96.

\section{5,8-Dihydroxy-2-methyl-2-(4-methyl-pent-3-enyl)-2H,6H-pyrano[3,2-b]xanthen-}

6-one (7c). Compound 7c was prepared similarly using 6 (250 mg, $1.02 \mathrm{mmol}$ ) and citral (0.90 mL, 5.12 mmol). 7c: Faint yellow solid (310 mg, 81\%); mp 195-196 ${ }^{\circ} \mathrm{C} ;{ }^{1} \mathrm{H}$ NMR (DMSO-d, $400 \mathrm{MHz}) \delta 1.39$ (s, 3H), 1.49 (s, 3H), 1.58 (s, 3H), 1.60-1.75 (m, 2H), 2.01 (q, $J=7.5 \mathrm{~Hz}, 2 \mathrm{H}), 5.04(\mathrm{t}, J=7.1 \mathrm{~Hz}, 1 \mathrm{H}), 5.69(\mathrm{~d}, J=10.2 \mathrm{~Hz}, 1 \mathrm{H}), 6.34(\mathrm{~s}, 1 \mathrm{H})$, $6.62(\mathrm{~d}, J=10.1 \mathrm{~Hz}, 1 \mathrm{H}), 7.27(\mathrm{~d}, J=8.0 \mathrm{~Hz}, 1 \mathrm{H}), 7.38(\mathrm{~s}, 1 \mathrm{H}), 7.43(\mathrm{~d}, J=9.1 \mathrm{~Hz}$, 1H), 10.03 (s, 1H), 13.23 (s, 1H); ${ }^{13} \mathrm{C}$ NMR (DMSO-d, $\left.100 \mathrm{MHz}\right) \delta 17.6,22.4,25.6$, 27.0, 41.2, 80.9, 94.5, 102.8, 103.6, 108.1, 115.2, 119.2, 120.5, 123.9, 124.9, 127.3, 131.3, 149.2, 154.3, 156.8, 156.9, 160.6, 180.2; MS (m/z) 379, 301, 267, 239, 217, 187 , 154, 126; IR (Nujol) 3400, 1643, 1612, 1584, $1464 \mathrm{~cm}^{-1}$. Anal. Calcd for $\mathrm{C}_{23} \mathrm{H}_{22} \mathrm{O}_{5}$ : C, 73.00; H, 5.86. Found: C, 73.11; H, 6.00. 
6,9-Dihydroxy-3-methyl-3H,7H-pyrano[2,3-c]xanthen-7-one (8b). Compound 8b was prepared similarly using 6 (250 mg, $1.02 \mathrm{mmol}$ ) and crotonaldehyde (0.80 mL, 10.24 mmol). 8b: Yellow solid (260 mg, 86\%); mp 139-141 ${ }^{\circ} \mathrm{C} ;{ }^{1} \mathrm{H}$ NMR (DMSO-d $d_{6}, 200$ MHz) $\delta 1.38(\mathrm{~d}, J=6.6 \mathrm{~Hz}, 3 \mathrm{H}), 5.08(\mathrm{~m}, 1 \mathrm{H}), 5.70(\mathrm{~d}, J=10.1 \mathrm{~Hz}, 1 \mathrm{H}), 6.07(\mathrm{~s}, 1 \mathrm{H})$, $6.70(\mathrm{~d}, J=9.5 \mathrm{~Hz}, 1 \mathrm{H}), 7.25(\mathrm{dd}, J=10.2 \& 2.0 \mathrm{~Hz}, 1 \mathrm{H}), 7.33(\mathrm{~d}, J=1.4 \mathrm{~Hz}, 1 \mathrm{H})$, $7.40(\mathrm{~d}, J=8.7 \mathrm{~Hz}, 1 \mathrm{H}), 10.01(\mathrm{~s}, 1 \mathrm{H}) ;{ }^{13} \mathrm{C}$ NMR (DMSO-d, $\left.50 \mathrm{MHz}\right) \delta 21.5,72.8$, 98.1, 101.0, 102.9, 108.1, 115.7, 119.2, 120.4, 123.8, 124.8, 149.1, 151.3, 154.3, 160.6, 162.4, 180.2; MS (m/z) 297, 283, 266, 239, 214, 206, 192, 154, 137, 126; IR (Nujol) 3335, 1653, 1578, 1464, $1377 \mathrm{~cm}^{-1}$. Anal. Calcd for $\mathrm{C}_{17} \mathrm{H}_{12} \mathrm{O}_{5}$ : C, 68.92; H, 4.08. Found: C, 68.86; H, 4.13.

\section{6,9-Dihydroxy-3-methyl-3-(4-methyl-pent-3-enyl)-3H,7H-pyrano[2,3-c]xanthen-}

7-one (8c). Compound 8c was prepared similarly using 6 (250 mg, $1.02 \mathrm{mmol}$ ) and citral (1.75 mL, $10.24 \mathrm{mmol}) .8 \mathrm{c}:$ Yellow oil (309 mg, 80\%); ${ }^{1} \mathrm{H} \mathrm{NMR}\left(\mathrm{CDCl}_{3}, 400 \mathrm{MHz}\right) \delta$ 1.49 (s, 3H), 1.60 (s, 3H), 1.67 (s, 3H), 1.68-1.77 (m, 1H), 1.78-1.88 (m, 1H), 2.15 (q, J = 7.6 Hz, 2H), 5.12 (t, $J=6.5 \mathrm{~Hz}, 1 \mathrm{H}), 5.54$ (d, $J=10.1 \mathrm{~Hz}, 1 \mathrm{H}), 6.21(\mathrm{~s}, 1 \mathrm{H}), 6.74$ (d, $J$ = $10.0 \mathrm{~Hz}, 1 \mathrm{H}), 6.99(\mathrm{~d}, J=9.1 \mathrm{~Hz}, 1 \mathrm{H}), 7.17$ (d, $J=8.0 \mathrm{~Hz}, 1 \mathrm{H}), 7.50(\mathrm{~s}, 1 \mathrm{H}), 7.88$ (bs, 1H), 12.54 (s, $1 \mathrm{H}) ;{ }^{13} \mathrm{C} \mathrm{NMR}\left(\mathrm{CDCl}_{3}, 100 \mathrm{MHz}\right) \delta 17.6,22.6,25.6,27.1,41.6,80.8$, 98.9, 100.6, 103.1, 108.3, 115.5, 118.7, 120.2, 123.6, 124.7, 125.7, 132.1, 149.8, 151.7, 153.2, 161.4, 162.8, 180.4; MS (m/z) 379, 344, 302, 265, 241, 220, 141; IR $\left(\mathrm{CHCl}_{3}\right)$ 3331, 1651, $1645 \mathrm{~cm}^{-1}$. Anal. Calcd for $\mathrm{C}_{23} \mathrm{H}_{22} \mathrm{O}_{5}$ : C, 73.00; H, 5.86. Found: C, 73.20; H, 5.72 . 